\title{
Assessment of the structured clinical interview (SCID) for DSM-5 for somatic symptom disorder in general hospital outpatient clinics in China
}

Yinan Jiang ${ }^{1}$, Jing Wei ${ }^{1 *}$ (D, Kurt Fritzsche ${ }^{2}$, Anne Christin Toussaint ${ }^{3}, \mathrm{Tao}_{\mathrm{Li}}{ }^{1}$, Jinya Cao ${ }^{1}$, Lan Zhang ${ }^{4}$, Yaoyin Zhang ${ }^{5}$, Hua Chen ${ }^{6}$, Heng $\mathrm{Wu}^{7}$, Xiquan $\mathrm{Ma}^{8}$, Wentian $\mathrm{Li}^{9}$, Jie Ren ${ }^{10}$, Wei $\mathrm{Lu}^{11}$ and Rainer Leonhart ${ }^{12}$

\begin{abstract}
Background: It is still unknown whether the "Somatic symptom disorders (SSD) and related disorders" module of the Structured Clinical Interview for DSM-5, research version (SCID-5-RV), is valid in China. This study aimed to assess the SCID-5-RV for SSD in general hospital outpatient clinics in China.

Methods: This multicentre cross-sectional study was conducted in the outpatient clinics of nine tertiary hospitals in Beijing, Jincheng, Shanghai, Wuhan, and Chengdu between May 2016 and March 2017. The "SSD and related disorders" module of the SCID-5-RV was translated, reversed-translated, revised, and used by trained clinical researchers to make a diagnosis of SSD. Several standardized questionnaires measuring somatic symptom severity, emotional distress, and quality of life were compared with the SCID-5-RV.

Results: A total of 699 patients were recruited, and 236 were diagnosed with SSD. Of these patients, 46 had mild SSD, 78 had moderate SSD, 100 had severe SSD, and 12 were excluded due to incomplete data. The SCID-5-RV for SSD was highly correlated with somatic symptom severity, emotional distress, and quality of life (all $P<0.001$ ) and could distinguish nonsevere forms of SSD from severe ones.
\end{abstract}

Conclusions: This study suggests that SCID-5-RV for SSD can distinguish SSD from non-SSD patients and severe cases from nonsevere cases. It has good discriminative validity and reflects the DSM-5 diagnostic approach that emphasizes excessive emotional, thinking, and behavioural responses related to symptoms.

Keywords: DSM-5, Somatization, Psychological tests, Validation

\section{Background}

Somatic symptom disorder (SSD), formerly known as somatoform disorder (SFD) [1], is one of the most common reasons for visiting physicians [2]. SSD includes somatic symptoms that are not associated with other mental

\footnotetext{
* Correspondence: weijing@pumch.cn

1 Department of Psychological Medicine, Peking Union Medical College Hospital, Chinese Academy of Medical Sciences \& Peking Union Medical College, Beijing, China

Full list of author information is available at the end of the article
}

disorders or cannot be medically explained in relation to a patient's general medical condition [2]. SSD has tremendous relevance for health care systems. Therefore, somatoform symptoms must be reliably classified to improve detection, adequate treatment, and relevant research efforts. In this study, the practical application of structured clinical interviews in this field will be explored.

SFD was introduced in the Diagnostic and Statistical Manual of Mental Disorders-Third Edition (DSM-III) [3] and modified for the DSM-IV [1], but researchers

(c) The Author(s). 2021 Open Access This article is licensed under a Creative Commons Attribution 4.0 International License, which permits use, sharing, adaptation, distribution and reproduction in any medium or format, as long as you give appropriate credit to the original author(s) and the source, provide a link to the Creative Commons licence, and indicate if changes were made. The images or other third party material in this article are included in the article's Creative Commons licence, unless indicated otherwise in a credit line to the material. If material is not included in the article's Creative Commons licence and your intended use is not permitted by statutory regulation or exceeds the permitted use, you will need to obtain permission directly from the copyright holder. To view a copy of this licence, visit http://creativecommons.org/licenses/by/4.0/. The Creative Commons Public Domain Dedication waiver (http://creativecommons.org/publicdomain/zero/1.0/) applies to the data made available in this article, unless otherwise stated in a credit line to the data. 
generally agreed that the definition of SFD needed substantial revision [4]. The current diagnostic DSM-5 criteria for SSD encompass the former diagnoses of SFD, pain disorder, undifferentiated somatoform disorder, and, in part, hypochondriasis. Compared with DSM-IV, DSM-5 introduced two major changes. First, the somatic symptoms criterion is no longer limited to medically unexplained symptoms; this eliminates the difficult and subjective distinction between medically explained and unexplained symptoms and mind-body dualism $[5,6]$. Second, criterion B now includes positive psychological diagnostic criteria; this criterion now includes excessive thoughts, feelings, and behaviours that are related to the somatic symptoms experienced by the patient or associated with health concerns. For criterion B, the patients had to present at least one of the following: 1) disproportionate and persistent thoughts about symptoms, 2) persistently high anxiety about health or symptoms, and 3) excessive time and energy devoted to the symptoms. Third, according to criterion $\mathrm{C}$, the symptoms have to persist for at least 6 months. Patients with SSD are classified as mild SSD (at least one B criterion), moderate SSD (two or more B criteria), and severe (two or more B criteria plus criterion C).

The DSM system is a common nomenclature to describe psychopathology, but it has been criticized repeatedly $[7,8]$. The major critique is that the DSM conceptualizes disorders as categorical entities for which the individuals are dichotomized as being with or without the disorder, which leads to a risk of overmedicalization $[7,8]$. Indeed, multiple studies have shown that most psychopathologies are not categorical entities but are rather continuous or dimensional hybrids of dimensional and categorical constructs [9-12].

The structured clinical interview for DSM-5, research version (SCID-5-RV) is a guide for semi-structured interviews for the major DSM-5 diagnoses; the latest version was published by the American Psychiatric Association in 2015 in English [13]. It is still unknown whether the categorical and symptom severity dimensional constructs of the "Somatic symptom disorders and related disorders" module of SCID-5-RV are valid.

Therefore, the aim of this study was to assess the SCID-5-RV for SSD in general hospital outpatient clinics in China. This should provide some basis for the further validation of the SCID-5-RV approach and for its official translation in Chinese.

\section{Methods}

\section{Study design and settings}

This study is a secondary analysis of a dataset that was collected for a previous multicentre cross-sectional study that was conducted from May 2016 to March 2017 in outpatient clinics of nine tertiary hospitals in North,
North-Central, East, Central, and West China (Beijing, Jincheng, Shanghai, Wuhan, and Chengdu). The nine participating centres were authoritative centres in the field of psychiatry in China. The modern biomedical setting was represented by the neurology and gastroenterology departments, the Traditional Chinese Medicine (TCM) department represented the TCM setting, and the psychological medicine department represented the psychosomatic medical settings.

\section{Subjects}

A total of 220 patients were recruited from each of the three medical settings. The screening days at each centre were randomly determined. All patients who were admitted to one of the study departments were approached for participation in the study using an informational hand-out. An informed consent form was used to explain the aims of the study to the patients. The patients were fully informed that their participation was voluntary, that the data would be analysed anonymously and that there were no disadvantages in case of refusal to participate.

The inclusion criteria were as follows: 1) $\geq 18$ years old; 2) visiting for treatment (i.e., not only picking up a prescription); 3) able to read and write; and 4) signed the written consent form. The exclusion criteria were as follows: 1) language barrier; 2) limited writing skills; 3) cognitive impairment; 4) psychosis; or 5) acute suicidal ideation.

\section{Assessments}

All patients who provided written informed consent filled in questionnaires for general information and quantitative assessment of psychopathology.

In general information acquisition, smoking status was classified as never smoking, former smoker, and current smoker. Drinking status was classified as never drinking, drinking only socially, history of drinking but now abstinent, and currently drinking $>3$ days/week. Exercise was classified as $>2 \mathrm{~h} /$ day, $1-2 \mathrm{~h} /$ day, $<1 \mathrm{~h} /$ day, and no physical exercise. The self-evaluation of treatment satisfaction and efficacy was divided into six grades $(0-5$ points): 0 means "not satisfied with the treatment in the past 6 months at all" or "considered that the treatment in the past 6 months was completely failed", and 5 means "very satisfied with the treatment in the past 6 months" or "considered that the treatment in the past 6 months was very successful".

The quantitative assessment of psychopathology included the following: 1) the Patient Health Questionnaire 15 (PHQ-15) was used to assess the number and severity of somatic symptoms; 2) the Somatic Symptom Scale-8 (SSS-8) was used to assess the somatic symptom burden; 3) the Patient Health Questionnaire 9 (PHQ-9) 
was used to assess depression; 4) the General Anxiety Disorder-7 (GAD-7) was used to assess anxiety; 5) the Whiteley-7 was used to assess health-related anxiety (WI7); 6) the WHO Disability Assessment Schedule (WHODAS 2.0); 7) the Somatic Symptom Disorder B-criteria (SSD-12); 8) the 12-item short-form health survey (SF-12) was used to assess quality of life; 9) questions on health care utilization; and 10) sociodemographic data (age, sex, level of education, marital status, etc.).

The PHQ-15 was validated in Chinese for somatic symptoms $[14,15]$. The SSS- 8 is a self-rating scale used to quantify the somatic symptom burden of patients in the past week and has been validated in Chinese patients [16]. The severity of depression and generalized anxiety were assessed using the 9-item depression scale PHQ-9 and the 7-item GAD-7, respectively. The Chinese versions of the PHQ-9 and GAD-7 have been shown to valid and reliable [17-19]. Illness anxiety was evaluated using the 7-item WI-7 Chinese version [20]. Populationlevel and clinical practice health and disability were assessed using the Chinese version of the WHO-DAS 2.0 [21]. Patients' perception of their symptom-related thoughts, feelings, and behaviours was assessed using the SSD-12, which was developed based on the DSM-5 criteria [22]. The SF-12 captures information on healthrelated quality of life (QoL) and has been validated in Chinese [23, 24].

\section{Diagnostic interview \\ Translation of the SCID-5-RV}

Given that a Chinese version of the SCID-5-RV was not available at the time, we developed a research version of a semi-structured clinical interview to assess the diagnostic criteria of SSD. The interview was adapted to the English version of SCID-5, which is considered the gold standard measure for DSM diagnoses. The "Somatic Symptom Disorders and Related Disorders" module of the SCID-5-RV was purchased. Some of the authors of this manuscript (KF, A-M M, AT, TL) formed a translation team. In the process of translation, both the SCID-5 SSD standard and the ITC-Test Adaptation Guidelines (Version 2000) of the International Examination Board [25] are taken into account. The specific translation process is clearly explained in the corresponding literature [26]. A final version was accepted and agreed upon by all Chinese researchers after translations were discussed. The Chinese translation did not obtain approval from the APA; therefore, the present study is a preliminary study that establishes the basis for a future Chinese version.

\section{The interview processes}

All patients underwent an interview (SCID-5-RV) following the criteria of the DSM-5 for SSD. All the research assistants (psychiatrists and postgraduate medical students in psychiatry) were trained on how to administer the SCID-5-RV. The assistants worked under the direct supervision of attending psychiatrists with $>3$ years of experience (i.e., the clinical heads of psychosomatic medicine).

Based on the results of the SCID-5-RV, the patients could be diagnosed with SSD in the presence of criteria $A$ and $B$ [2]. Patients with one B criterion were classified as having mild SSD, those with at least two B criteria were classified as having moderate SSD, and those with at least two $B$ criteria and the $C$ criterion were classified as having SSD.

The results of the SCID-5-RV were compared with the results of the other scales. The combination of the PHQ-15 and SSD-12 has been used to diagnose SSD [27].

\section{Statistical analysis}

All data were stored at the University Medical Centre Freiburg. The same centre was responsible for monitoring the project sites and for data analysis. The study was approved by the ethics committees of all participating centres. The approval number at the University Medical Centre Freiburg was S-K276. The data were collected by research assistants between May 2016 and March 2017.

All statistical analyses were performed using SPSS 22.0 (IBM, Armonk, NY, USA). Categorical variables are described as numbers and percentages and were analysed using the chi-square test. Continuous variables are presented as the means and standard deviations and were analysed using Student's independent-samples t-test. One-way analysis of variance and the LSD post hoc test were used for the comparison of continuous variables among multiple groups. Spearman's correlation analysis was used to examine the relationship between the SCID5-RV and the other scales. Two-tailed $P$-values $<0.05$ were considered significant. Alpha inflation might be an issue, and to mitigate it, only P-values $<0.001$ were considered high-impact differences or correlations.

\section{Results \\ Enrolment.}

During the study period, 1269 patients were contacted, and 699 (55.1\%) were enrolled. All 699 completed the questionnaires and clinical interviews. Among those who did not participate in the study, 68 (5.4\%) met at least one exclusion criterion, and 502 (39.6\%) refused to participate $(53.0 \%$ reported having no time to participate; $29.5 \%$ reported having no interest in the study; $8.4 \%$ reported a lack of trust in the interviewers; $6.8 \%$ reported not feeling well enough to participate; and $2.4 \%$ provided other reasons). Among them, 150 were from Peking Union Medical College Hospital, 50 were from Beijing 
Hospital of Traditional Chinese Medicine, Capital University, 158 were from West China Hospital, Sichuan University, 53 were from Sichuan Provincial People's Hospital, 50 were from Tongji Hospital of Tongji University, 50 were from Dongfang Hospital of Tongji University, 55 were from Zhongshan Hospital Affiliated to Fudan University, 52 were from Renmin Hospital of Wuhan University, and 81 were from Jincheng People's Hospital. Two patients had incomplete data and were excluded, and 697 patients finally underwent diagnostic interviews [26].

\section{Diagnosis of SSD.}

According to the SCID-5-RV results, 236 (33.8\%) were diagnosed with SSD. Of these, 46 (19.5\%) had mild SSD, 78 (33.1\%) had moderate SSD, 100 (42.4\%) had severe SSD, and $12(5.1 \%)$ were excluded due to incomplete data (Fig. 1).

\section{Sociodemographic characteristics.}

When comparing the patients with and without SSD, there were no significant differences between the two groups in demographic variables. The patients with SSD were separated according to severity, and there were no differences among the three groups (all $P>0.05$ ) (Table 1).

\section{Clinical characteristics.}

When comparing the patients with and without SSD, the patients with SSD had lower treatment satisfaction $(2.5 \pm 1.8$ vs. $3.3 \pm 1.6, \quad P<0.001)$ and worse selfevaluation of the treatment effect $(2.4 \pm 1.6$ vs. $3.1 \pm 1.6$, $\mathrm{P}<0.001)$. The scores of the questionnaires for symptom severity, emotional distress, and SSD B-criteria in the SSD group were higher than those in the non-SSD group, while the scores for quality of life in the SSD

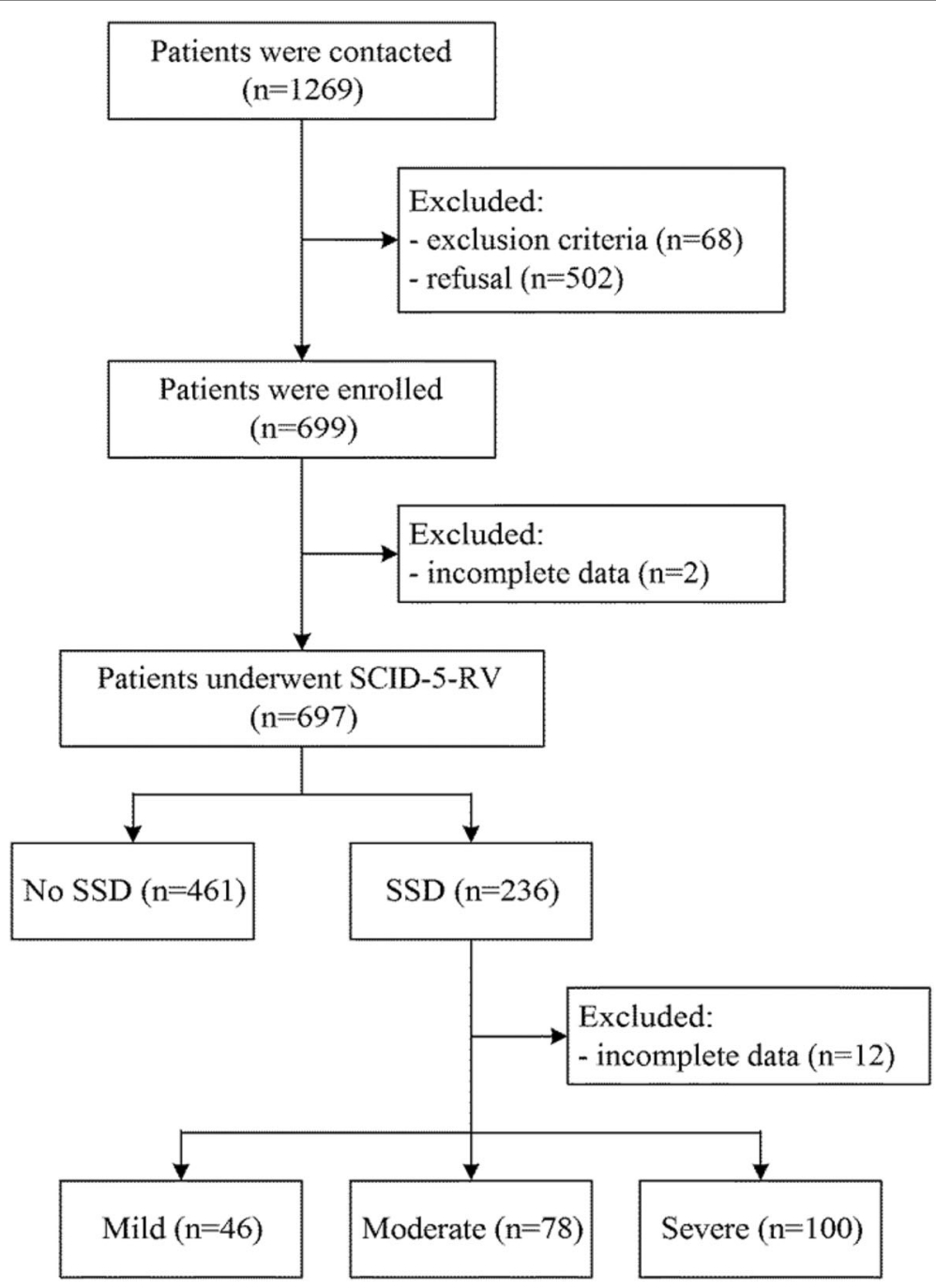

Fig. 1 Patient flowchart. SCID-5-RV: Structured clinical interview for DSM-5, research version; SSD: Somatic Symptom Disorder 
Table 1 Sociodemographic characteristics of the patients

\begin{tabular}{|c|c|c|c|c|c|c|c|}
\hline & No SSD $(n=473)$ & SSD $(n=224)$ & $\mathbf{P}$ & Mild $(n=46)$ & Moderate $(n=78)$ & Severe $(n=100)$ & $\mathbf{P}$ \\
\hline Age (years) & $43.1 \pm 14.7$ & $43.0 \pm 14.0$ & 0.957 & $43.5 \pm 14.7$ & $40.2 \pm 14.2$ & $45.4 \pm 13.1$ & 0.052 \\
\hline Sex, female (\%) & $284(61.6 \%)$ & $143(60.6 \%)$ & 0.806 & $21(45.7 \%)$ & $50(64.1 \%)$ & $63(63.0 \%)$ & 0.088 \\
\hline Health insurance, yes (\%) & $400(87.7 \%)$ & $200(85.5 \%)$ & 0.406 & $38(82.6 \%)$ & $66(84.6 \%)$ & 85 (86.7\%) & 0.800 \\
\hline Residence, urban (\%) & $386(83.7 \%)$ & 187(79.6\%) & 0.174 & $35(76.1 \%)$ & $60(76.9 \%)$ & $84(84.8 \%)$ & 0.306 \\
\hline Marital status, married (\%) & $343(74.4 \%)$ & $163(69.1 \%)$ & 0.194 & $31(67.4 \%)$ & $49(62.8 \%)$ & $77(77.0 \%)$ & 0.364 \\
\hline Family income (monthly, Yuans) (\%) & & & 0.196 & & & & 0.850 \\
\hline Low $(<4000)$ & $145(31.7 \%)$ & $89(38.0 \%)$ & & $17(37.0 \%)$ & $32(41.6 \%)$ & $36(36.4 \%)$ & \\
\hline Middle (4000-8000) & 162(35.4\%) & $80(34.2 \%)$ & & $15(32.6 \%)$ & $24(31.2 \%)$ & $38(38.4 \%)$ & \\
\hline High (> 8000) & $151(33.0 \%)$ & $65(27.8 \%)$ & & $14(30.4 \%)$ & $21(27.3 \%)$ & $25(25.3 \%)$ & \\
\hline Profession, employed (\%) & $237(51.4 \%)$ & 105(44.5\%) & 0.215 & $25(54.3 \%)$ & $34(43.6 \%)$ & 39(39.0\%) & 0.699 \\
\hline Education, university or higher (\%) & $230(49.9 \%)$ & $105(44.5 \%)$ & 0.227 & $20(43.5 \%)$ & $41(52.6 \%)$ & $38(38.0 \%)$ & 0.239 \\
\hline Exercise in winter, never (\%) & $123(26.7 \%)$ & $70(29.7 \%)$ & 0.670 & $15(32.6 \%)$ & $20(25.6 \%)$ & $33(33.0 \%)$ & 0.680 \\
\hline Exercise in summer, never (\%) & $95(20.6 \%)$ & $54(22.9 \%)$ & 0.597 & $9(19.6 \%)$ & $14(17.9 \%)$ & $29(29.0 \%)$ & 0.113 \\
\hline Smoking, never (\%) & $340(73.8 \%)$ & $165(70.2 \%)$ & 0.473 & $31(67.4 \%)$ & $58(74.4 \%)$ & $67(67.7 \%)$ & 0.165 \\
\hline Alcohol, never (\%) & $218(47.3 \%)$ & $127(54 \%)$ & 0.385 & $26(56.5 \%)$ & 39 (50.0\%) & $54(54.5 \%)$ & 0.908 \\
\hline
\end{tabular}

SSD: somatic symptom disorders

group were significantly lower than those in the nonSSD group (all $P<0.05$ ) (Table 2).

When comparing the mild, moderate, and severe groups, the SSD-12 scores and numbers of visits to doctors in the last 12 months were significantly different among the three groups. The WHO DAS 2.0, PHQ-9, GAD-7, and WI-7 scores indicated that the mild and moderate groups had significantly lower scores than the severe group, but there was no significant difference between the mild and moderate groups (all $P<0.05$ ) (Table 2). The PHQ-15 and SSS-8 scores were significantly lower in the moderate group than in the severe group.
The SF-12 PCS and MCS scores indicated that the scores of the mild group were significantly lower than those of the severe group (all $\mathrm{P}<0.05)$ (Table 2).

\section{Spearman's correlation analysis.}

The results of the SCID-5-RV for SSD diagnosis were highly correlated with somatic symptom severity, emotional distress, and quality of life, which were assessed by the PHQ-15, SSS-8, PHQ-9, GAD-7, WI-7, SSD-12, WHO-DAS 2.0, SF-12 PCS, and SF-12 MCS (all $P<$ 0.001). The SCID-5-RV for SSD severity was significantly correlated with scores on the PHQ-9, GAD-7, WI-7,

Table 2 Clinical characteristics of the patients

\begin{tabular}{|c|c|c|c|c|c|c|c|}
\hline & No SSD $(n=473)$ & SSD $(n=224)$ & $\mathbf{P}$ & Mild $(n=46)$ & Moderate $(n=78)$ & Severe $(n=100)$ & $\mathbf{P}$ \\
\hline Satisfaction & $3.3 \pm 1.6$ & $2.5 \pm 1.8$ & $<0.001$ & $2.7 \pm 1.8$ & $2.2 \pm 1.7$ & $2.5 \pm 1.9$ & 0.344 \\
\hline Effect & $3.1 \pm 1.6$ & $2.4 \pm 1.6$ & $<0.001$ & $2.6 \pm 1.6$ & $2.2 \pm 1.5$ & $2.4 \pm 1.7$ & 0.366 \\
\hline PHQ-15 & $8.0 \pm 4.8$ & $12.0 \pm 5.5$ & $<0.001$ & $12.0 \pm 6.1$ & $10.7 \pm 5.0$ & $13.1 \pm 5.6$ & $0.019^{c}$ \\
\hline WHO DAS 2.0 & $17.3 \pm 5.8$ & $22.7 \pm 8.5$ & $<0.001$ & $21.3 \pm 8.0$ & $21.1 \pm 6.8$ & $24.8 \pm 9.8$ & $0.006^{b, c}$ \\
\hline PHQ-9 & $6.8 \pm 5.8$ & $11.8 \pm 6.8$ & $<0.001$ & $10.4 \pm 7.9$ & $11.0 \pm 6.0$ & $13.4 \pm 6.8$ & $0.012^{b, c}$ \\
\hline GAD-7 & $5.1 \pm 5.2$ & $9.7 \pm 6.1$ & $<0.001$ & $7.8 \pm 6.3$ & $9.0 \pm 5.6$ & $11.1 \pm 6.3$ & $0.004^{b, c}$ \\
\hline WI-7 & $13.2 \pm 5.5$ & $21.4 \pm 7.4$ & $<0.001$ & $18.6 \pm 7.3$ & $20.7 \pm 6.9$ & $23.3 \pm 7.2$ & $0.001^{b, c}$ \\
\hline SSD-12 & $9.1 \pm 9.4$ & $23.6 \pm 11.4$ & $<0.001$ & $17.8 \pm 10.8$ & $22.1 \pm 10.2$ & $27.5 \pm 10.9$ & $<0.001^{\mathrm{a}, \mathrm{b}, \mathrm{c}}$ \\
\hline SSS-8 & $7.0 \pm 5.1$ & $12.0 \pm 6.5$ & $<0.001$ & $11.6 \pm 7.0$ & $10.8 \pm 5.3$ & $13.3 \pm 7.0$ & $0.034^{c}$ \\
\hline SF-12 (PCS) & $45.1 \pm 8.6$ & $39.1 \pm 9.0$ & $<0.001$ & $41.0 \pm 8.2$ & $40.0 \pm 9.0$ & $37.4 \pm 9.0$ & $0.038^{b}$ \\
\hline SF-12 (MCS) & $44.6 \pm 11.5$ & $34.9 \pm 11.3$ & $<0.001$ & $38.6 \pm 13.9$ & $34.4 \pm 11.6$ & $33.5 \pm 9.8$ & $0.044^{b}$ \\
\hline Number of visits & $1.8 \pm 0.9$ & $2.2 \pm 1.1$ & $<0.001$ & $1.9 \pm 1.0$ & $1.9 \pm 1.0$ & $2.6 \pm 1.0$ & $<0.001^{\mathrm{a}, \mathrm{b}, \mathrm{c}}$ \\
\hline
\end{tabular}

Post hoc analysis was adjusted by LSD. a: $\mathrm{P}<0.05$ Mild vs. Moderate; $\mathrm{b}: \mathrm{P}<0.05$ Mild vs. Severe; $\mathrm{C}$ P $<0.05$ Moderate vs. Severe

PHQ-15: Patient Health Questionnaire 15; WHO-DAS 2.0: WHO Disability Assessment Schedule; PHQ-9: Patient Health Questionnaire 9; GAD-7: General Anxiety Disorder-7; WI-7: Whiteley-7 for health-related anxiety; SSD-12: Somatic Symptom Disorder B-criteria; SSS-8: Somatic Symptom Scale-8; SF-12: 12-item short-form health survey; PCS: physical component score; MCS: mental component score; SCID-5-RV: Structured clinical interview for DSM-5, research version 
SSD-12, WHO-DAS 2.0, SF-12 PCS, and SF-12 MCS (all $\mathrm{P}<0.05$ ) (Table 3).

\section{Discussion}

It is still unknown whether the "SSD and related disorders" module of SCID-5-RV is valid. Therefore, this study aimed to assess the SCID-5-RV for SSD in general hospital outpatient clinics in China. The results suggest that SCID-5-RV for SSD can distinguish SSD from nonSSD patients and severe forms of SSD from nonsevere forms. It has good discriminative validity with other tools and reflects the DSM-5 diagnostic approach that emphasizes excessive emotional, thinking, and behavioural responses related to symptoms.

There were no significant differences in sociodemographic and lifestyle variables between the SSD and nonSSD groups or among the severity groups. These results suggest that sociodemographic factors did not affect the potential diagnostic value of the SCID-5-RV.

In the present study, the SCID-5-RV could identify patients with SSD. A study in China showed that using the SCID-5 led to a frequency of $36.5 \%$ for SSD but that there were differences in sociodemographic characteristics among SSD severity groups, with patients with mild SSD having better socioeconomic conditions than those with severe SSD [28]. A Turkish version of the SCID-5$\mathrm{CV}$ showed a $\mathrm{k}$ of 0.65 for SDD [29]. Many questionnaires can be used for the evaluation of $\operatorname{SSD}[5,6]$, but it is considered that the PHQ-15, SSS- 8 , and SSD-12 provide good diagnostic potential [27]. In the present study, the Spearman correlation of the SCID-5-RV was the strongest with SSD-12, but it was also correlated with PHQ-15 and SSS-8. These results suggest that the Chinese version of the SCID-5-RV could be used for the

Table 3 Spearman correlation between SCID-5-RV and other assessment scales

\begin{tabular}{llllll}
\hline & \multicolumn{2}{l}{ Diagnostic $(\boldsymbol{n}=6 \mathbf{6 9 7})$} & & \multicolumn{2}{l}{ Severity $(\mathbf{n}=\mathbf{2 2 4})$} \\
\cline { 2 - 3 } \cline { 6 - 7 } & $\mathbf{r}$ & $\mathbf{P}$ & & $\mathbf{r}$ & $\mathbf{P}$ \\
\hline PHQ-15 & 0.355 & $<0.001$ & & 0.112 & 0.095 \\
SSS-8 & 0.385 & $<0.001$ & & 0.130 & 0.053 \\
PHQ-9 & 0.366 & $<0.001$ & & 0.189 & 0.005 \\
GAD-7 & 0.370 & $<0.001$ & & 0.217 & 0.001 \\
WI-7 & 0.530 & $<0.001$ & & 0.254 & $<0.001$ \\
SSD-12 & 0.563 & $<0.001$ & & 0.339 & $<0.001$ \\
WHO DAS 2.0 & 0.351 & $<0.001$ & & 0.182 & 0.006 \\
SF-12 (PCS) & -0.310 & $<0.001$ & & -0.166 & 0.013 \\
SF-12 (MCS) & -0.375 & $<0.001$ & & -0.154 & 0.022
\end{tabular}

PHQ-15: Patient Health Questionnaire15; SSS-8: Somatic Symptom Scale-8; PHQ-9: Patient Health Questionnaire 9;GAD-7: General Anxiety Disorder-7;WI-7: Whiteley-7 for health-related anxiety; SSD-12: Somatic Symptom Disorder Bcriteria; WHO-DAS 2.0: WHO Disability Assessment Schedule; SF-12: the 12item Short Form Health Survey; PCS: physical composite score; MCS: mental composite score diagnosis of SSD in Chinese patients. As a diagnostic tool, SCID-5-RV for SSD only has a moderate positive correlation with SSD-12 and WI-7 and a low correlation with other criterion tools such as PHQ-9 and GAD-7. This finding is plausible considering that SCID-5-RV for SSD could distinguish somatic disorders from anxiety and depression disorders well and is more influenced by thoughts, emotions, and behaviours related to symptoms. Similarly, there was a weak correlation between the PHQ-15 and SSS-8 scores. This result may reflect that, under the new diagnostic criteria, the number and load of somatic symptoms have a weaker impact on the diagnostic results.

The PHQ-15, SSS-8, and SSD-12 have also been shown to be able to discriminate among SSD severity groups [27]. Among patients with mild, moderate, and severe SSD, there were significant differences in the SSD-12 scores and the number of doctor visits in the past 12 months. This suggests that with the increase in the severity of SSD, patients have gradually aggravated emotional, thinking, and behavioural problems related to the symptoms and gradually increased use of medical resources. The WHO-DAS 2.0, PHQ-9, GAD-7, and WI-7 scores indicated that the mild and moderate groups were significantly lower than the severe group. This indicates that patients in the severe group have more severe functional impairment, disease belief, anxiety, and depression than those in the nonsevere group. The PHQ-15 and SSS-8 scores indicated that the scores of the moderate group were significantly lower than those of the severe group. This indicates that the somatic symptom load of the severe group was heavier than that of the moderate group. The PCS and MCS scores on the SF-12 indicated that the scores of the mild group were significantly higher than those of the severe group. This indicates that the severe group has more severe functional impairment than the mild group.

The strength of this study was that the outpatient clinics of four departments in nine tertiary hospitals in five Chinese cities participated in this study, resulting in a large sample. Nevertheless, this study had limitations. This study used the convenient sampling method, which may have led to selection bias. This study was crosssectional and thus lacked follow-up observations of patients to understand the outcome of the disease. The diagnostic value of the SCID-5-RV was not verified. Future studies should formally validate the SCID-5-RV Chinese version.

\section{Conclusion}

In conclusion, this study showed that the SCID-5-RV for SSD could well distinguish SSD patients from non-SSD patients and severe SSD patients from nonsevere SSD patients. There was no significant difference in the 
clinical characteristics between patients with mild SSD and those with moderate SSD. Both as a diagnostic tool and severity assessment tool, the SSD module of the SCID-5-RV Chinese version had good discriminative validity with other criterion tools, including the PHQ-15, SSS-8, PHQ-9, GAD-7, WI-7, SSD-12, WHO DAS 2.0, and SF-12. Therefore, it is necessary to further explore an evaluation tool suitable for the clinical application of the SSD module of SCID-5-RV in the future.

\section{Acknowledgements}

Not Applicable.

\section{Authors' contributions}

YJ was the project leader; she was responsible for the organization of data collection and drafted the manuscript. JW made substantial contributions to the study conception and design. KF made substantial contributions to the study conception and design and to data analysis and interpretation and drafted the manuscript. AT made substantial contributions to the study conception and design and helped to draft the manuscript and critically revise the text for important intellectual content. TL and JC helped organize the data collection and draft the manuscript. LZ, YZ, HC, HW, XM, WL, JR, and WL participated in its design and coordination and helped draft the manuscript. RL participated in the study design and performed the statistical analysis. All authors read and improved the final manuscript.

\section{Funding}

Not Applicable.

\section{Availability of data and materials}

The dataset supporting the conclusions of this article is included within the article.

\section{Ethics approval and consent to participate}

The authors assert that all procedures contributing to this work comply with the ethical standards of the relevant national and institutional committees on human experimentation and with the 1975 Declaration of Helsinki, as revised in 2008. All procedures involving human participants/patients were approved by the institutional review board of the University of Freiburg registered under the number 494/17 and the institutional review board of Peking Union Medical College Hospital registered under the number S-K276. All patients who provided written informed consent filled in questionnaires for general information and quantitative assessment of psychopathology.

\section{Consent for publication}

Not Applicable.

\section{Competing interests}

All authors declare that they have no competing interests.

\section{Author details}

'Department of Psychological Medicine, Peking Union Medical College Hospital, Chinese Academy of Medical Sciences \& Peking Union Medical College, Beijing, China. ${ }^{2}$ Department of Psychosomatic Medicine and Psychotherapy, Medical Center - University of Freiburg, Faculty of Medicine, Freiburg im Breisgau, Germany. ${ }^{3}$ Department of Psychosomatic Medicine and Psychotherapy, University Medical Center Hamburg-Eppendorf, Hamburg, Germany. ${ }^{4}$ Mental Health Centre, West China Hospital, Sichuan University, Chengdu, China. ${ }^{5}$ Department of Psychosomatic Medicine, Sichuan Provincial People's Hospital, University of Electronic Science and Technology of China, Chengdu, China. ${ }^{6}$ Department of Psychological Medicine, Zhong Shan Hospital, Fudan University, Shanghai, China. ${ }^{7}$ Department of Psychosomatic Medicine, Tongji Hospital, School of Medicine, Tongji University, Shanghai, China. ${ }^{8}$ Department of Psychosomatic Medicine, Dongfang Hospital, School of Medicine, Tongji University, Shanghai, China. ${ }^{9}$ Department of Clinic Psychology, Wuhan Mental Health Center, Wuhan, China. ${ }^{10}$ Department of Rehabilitation, General Hospital of Jincheng Anthracite Coal Mining Group Co. Ltd, Jincheng, China. "'Department of Psychosomatic Medicine, Beijing Hospital of Traditional Chinese Medicine, Capital University, Beijing, China.
${ }^{12}$ Institute of Psychology, University of Freiburg, Freiburg im Breisgau, Germany.

Received: 3 August 2020 Accepted: 17 February 2021

Published online: 10 March 2021

\section{References}

1. American Psychiatric Association. Diagnostic and statistical manual of mental disorders (DSM-4 ${ }^{\oplus}$ ). 4th ed. Washington, D.C.: American Psychiatric Association Publishing; 1994.

2. American Psychiatric Association. Diagnostic and statistical manual of mental disorders (DSM-5 ${ }^{\oplus}$ ). 5th ed. Washington, D.C.: American Psychiatric Association Publishing: 2013.

3. American Psychiatric Association. Diagnostic and statistical manual of mental disorders (DSM-3 ${ }^{\oplus}$ ). 3rd ed. Washington, D.C.: American Psychiatric Association Publishing; 1980.

4. Rief W, Martin A. How to use the new DSM-5 somatic symptom disorder diagnosis in research and practice: a critical evaluation and a proposal for modifications. Annu Rev Clin Psychol. 2014;10:339-67.

5. Henningsen P. Management of somatic symptom disorder. Dialogues Clin Neurosci 2018;20(1):23-31. PubMed PMID: 29946208; PubMed Central PMCI D: PMC6016049.

6. D'Souza RS, Hooten WM. Somatic Syndrome Disorders. 2020 Jul 10. In: StatPearls [Internet]. Treasure Island (FL): StatPearls Publishing; 2020 Jan-. PMID: 30335286

7. Pickersgill MD. Debating DSM-5: diagnosis and the sociology of critique. J Med Ethics 2014;40(8):521-525. doi: https://doi.org/10.1136/medethics-2 013-101762. PubMed PMID: 24327375; PubMed Central PMCID: PMC4112449.

8. Watts G. Critics attack DSM-5 for overmedicalising normal human behaviour. Bmj. 2012;344:e1020. doi: https://doi.org/10.1136/bmj.e1020. PubMed PMID: 22327355.

9. Krueger RF, Markon KE. Understanding psychopathology: melding behavior genetics, personality, and quantitative psychology to develop an empirically based model. Curr Dir Psychol Sci 2006;15(3):113-117. doi: https://doi.org/1 0.1111/j.0963-7214.2006.00418.x. PubMed PMID: 18392116; PubMed Central PMCID: PMC2288576.

10. Wright AG, Krueger RF, Hobbs MJ, Markon KE, Eaton NR, Slade T. The structure of psychopathology: toward an expanded quantitative empirical model. J Abnorm Psychol 2013;122(1):281-294. doi: https://doi.org/10.103 7/a0030133. PubMed PMID: 23067258; PubMed Central PMCID: PMC3570590.

11. Borsboom D, Rhemtulla M, Cramer AO, van der Maas HL, Scheffer M, Dolan CV. Kinds versus continua: a review of psychometric approaches to uncover the structure of psychiatric constructs. Psychol Med 2016;46(8):1567-1579. doi: https://doi.org/10.1017/S0033291715001944. PubMed PMID: 26997244

12. Eaton NR, Krueger RF, Docherty AR, Sponheim SR. Toward a model-based approach to the clinical assessment of personality psychopathology. J Pers Assess 2014;96(3):283-292. doi: https://doi.org/10.1080/00223891.2013.8302 63. PubMed PMID: 24007309; PubMed Central PMCID: PMC4353810.

13. American Psychiatric Association. Structured clinical interview for DSM-5, research version (SCID-5-RV). Washington, D.C.: American Psychiatric Association Publishing; 2015

14. Zhang L, Fritzsche K, Liu Y, Wang J, Huang M, Wang Y, et al. Validation of the Chinese version of the PHQ-15 in a tertiary hospital. BMC psychiatry. 2016;16:89. doi: https://doi.org/10.1186/s12888-016-0798-5. PubMed PMID: 27044309; PubMed Central PMCID: PMC4820992.

15. Leonhart R, de Vroege L, Zhang L, Liu Y, Dong Z, Schaefert R, et al. Comparison of the Factor Structure of the Patient Health Questionnaire for Somatic Symptoms (PHQ-15) in Germany, the Netherlands, and China. A Transcultural Structural Equation Modeling (SEM) Study. Frontiers in psychiatry. 2018;9:240. doi: https://doi.org/10.3389/fpsyt.2018.00240. PubMed PMID: 29997528; PubMed Central PMCID: PMC6028697.

16. Jiang M, Zhang W, Su X, Gao C, Chen B, Feng Z, et al. Identifying and measuring the severity of somatic symptom disorder using the self-reported somatic symptom scale-China (SSS-CN): a research protocol for a diagnostic study. BMJ Open 2019;9(9):e024290. doi: https://doi.org/10.1136/bmjopen-2 018-024290. PubMed PMID: 31558447; PubMed Central PMCID: PMC6773285.

17. Wang W, Bian Q, Zhao Y, Li X, Wang W, Du J, et al. Reliability and validity of the Chinese version of the patient health questionnaire (PHQ-9) in the 
general population. Gen Hosp Psychiatry 2014;36(5):539-544. doi: https:// doi.org/10.1016/j.genhosppsych.2014.05.021. PubMed PMID: 25023953.

18. Tong X, An D, McGonigal A, Park SP, Zhou D. Validation of the generalized anxiety Disorder-7 (GAD-7) among Chinese people with epilepsy. Epilepsy Res 2016;120:31-36. doi: https://doi.org/10.1016/j.eplepsyres.2015.11.019. PubMed PMID: 26709880.

19. Xiong N, Fritzsche K, Wei J, Hong X, Leonhart R, Zhao X, et al. Validation of patient health questionnaire $(\mathrm{PHQ})$ for major depression in Chinese outpatients with multiple somatic symptoms: a multicenter cross-sectional study. J Affect Disord 2015;174:636-643. doi: https://doi.org/10.1016/j.jad.2 014.12.042. PubMed PMID: 25576931.

20. Lee S, Ng KL, Ma YL, Tsang A, Kwok KP. A general population study of the Chinese Whiteley-7 index in Hong Kong. J Psychosom Res 2011;71(6):387391. doi: https://doi.org/10.1016/j.jpsychores.2011.05.013. PubMed PMID: 22118380.

21. Chiu TY, Yen CF, Chou CH, Lin JD, Hwang AW, Liao HF, et al. Development of traditional Chinese version of World Health Organization disability assessment schedule 2.0 36-item (WHODAS 2.0) in Taiwan: validity and reliability analyses. Res Dev Disabil 2014;35(11):2812-2820. doi: https://doi. org/10.1016/j.ridd.2014.07.009. PubMed PMID: 25094056.

22. Wei J, Fritzsche K, Toussaint AC, Jiang Y, Cao J, Zhang L, et al. Validation of the Chinese version of somatic symptom disorder-B criteria scale (SSD-12) for detecting DSM-5 somatic symptom disorders: a multicenter study. Psychosom Med 2019; In Press.

23. Fong DY, Lam CL, Mak KK, Lo WS, Lai YK, Ho SY, et al. The short Form-12 health survey was a valid instrument in Chinese adolescents. J Clin Epidemiol 2010;63(9):1020-1029. doi: https://doi.org/10.1016/j.jclinepi.2 009.11.011. PubMed PMID: 20189764.

24. Lam CL, Tse EY, Gandek B. Is the standard SF-12 health survey valid and equivalent for a Chinese population? Quality of life research 2005;14(2):539547. doi: https://doi.org/10.1007/s11136-004-0704-3. PubMed PMID: 15892443.

25. Hambleton RK, Hambleton R, Merenda P, Spielberger C. Issues, designs, and technical guidelines for adapting tests into multiple languages and cultures. Adapting educational and psychological tests for cross-cultural assessment. Hillsdale, NJ: Erlbaum; 2005.

26. Cao J, Wei J, Fritzsche K, Toussaint AC, Li T, Jiang Y, Zhang L, Zhang Y, Chen H, Wu H, Ma X, Li W, Ren J, Lu W, Müller AM, Leonhart R. Prevalence of DSM-5 somatic symptom disorder in Chinese outpatients from general hospital care. Gen Hosp Psychiatry 2020;62:63-71. doi: https://doi.org/10.101 6/j.genhosppsych.2019.11.010. Epub 2019 Nov 29. PMID: 31841874.

27. Toussaint A, Husing P, Kohlmann S, Lowe B. Detecting DSM-5 somatic symptom disorder: criterion validity of the patient health Questionnaire-15 (PHQ-15) and the somatic symptom Scale-8 (SSS-8) in combination with the somatic symptom disorder - B criteria scale (SSD-12). Psychol Med 2019:110. doi: https://doi.org/10.1017/S003329171900014X. PubMed PMID: 30729902

28. Xiong $N$, Zhang $Y$, Wei J, Leonhart $R$, Fritzsche $K$, Mewes $R$, et al. Operationalization of diagnostic criteria of DSM-5 somatic symptom disorders. BMC psychiatry 2017;17(1):361. doi: https://doi.org/10.1186/s12 888-017-1526-5. PubMed PMID: 29115965; PubMed Central PMCID: PMC5678573.

29. Elbir M, Alp Topbas O, Bayad S, Kocabas T, Topak OZ, Cetin S, et al. [Adaptation and Reliability of the Structured Clinical Interview for DSM-5Disorders - Clinician Version (SCID-5/CV) to the Turkish Language]. Turk psikiyatri dergisi $=$ Turkish journal of psychiatry. 2019;30(1):51-6. PubMed PMID: 31170307.

\section{Publisher's Note}

Springer Nature remains neutral with regard to jurisdictional claims in published maps and institutional affiliations.

Ready to submit your research? Choose BMC and benefit from:

- fast, convenient online submission

- thorough peer review by experienced researchers in your field

- rapid publication on acceptance

- support for research data, including large and complex data types

- gold Open Access which fosters wider collaboration and increased citations

- maximum visibility for your research: over $100 \mathrm{M}$ website views per year

At BMC, research is always in progress.

Learn more biomedcentral.com/submissions 